UCRL $-\Upsilon-114373$

PREPRINT

\title{
Spectroscopy and Atomic Physics in EBIT and SuperEBIT
}

\author{
P. Beiersdorfer \\ M. Bitter \\ M. Chen \\ V. Decaux \\ S. Elliott \\ S. Kahn \\ D. Knapp \\ R. Marrs \\ A. Osterheld \\ D. Vogel \\ K. Widmann
}

Prepared for Submittal to

Proceedings 9th APS Topical Conference on Atomic Processes in Plasmas San Antonio, TX September 19-23, 1993

December 16, 1993

This in a preprint of a paper intended for publication in a journal or proceedings. Since changer mas be made before publication, this preprint is made available with the understanding that it will not be cited or reproduced without the permission of the author. 


\section{DISCLAIMER}

This document was prepared as an account of work sponsored by an agency of the United States Government. Neither the United States Government nor the University of California nor any of their employees, makes any warranty, express or implied, or assumes any legal liability or responsibility for the accuracy, completeness, or usefulness of any information, apparatus, product, or process disdosed, or represents that its use would not infringe privately owned rights. Reference herein to any specific commercial products, process, or service by trade name, trademark, manufacturer, or otherwise, does not necessarily constitute or imply its endorsement, recommendation, or favoring by the United States Government or the University of Californis. The views and opinions of authors expressed herein do not necessarily state or reflect those of the United States Government or the University of Califormia, and shall not be used for advertising or product endorsement purposes. 


\title{
Spectroscopy and Atomic Physics in EBIT and SuperEBIT
}

\author{
P. Beiersdorfer, M. Bitter, ${ }^{*}$ M. Chen, V. Decaux, S. Elliott, S. Kahn, ${ }^{\dagger}$ D. Knapp \\ R. Marrs, A. Osterheld, D. Vogel, $¥$ K. Widmann
}

Lawrence Livermore National Laboratory, Livermore, CA 94550

\begin{abstract}
An overview is given of $x$-ray measurements in progress on the Livermore electron beam ion trap facilities. The measurements include detailed investigations of the satellite spectrum of heliumlike krypton $\mathrm{Kr}^{34+}$ for diagnostic applications in future tokamak fusion reactors, precise measurements of the 2-2 transition energies in neonlike $\mathrm{U}^{82+}$ through lithiumlike $\mathrm{U}^{89+}$ to test the predictions of QED theory, and investigations of line formation by innershell ionization and non-resonant electron capture for the development of line diagnostics applicable to non-equilibrium plasmas.
\end{abstract}

\footnotetext{
${ }^{*}$ Princeton Plasma Physics Laboratory, Princeton, NJ 08543.

†Space Sciences Laboratory, Berkeley, CA 94720.

$\ddagger$ HQ ACC/DRAN, Langley Air Force Base, VA 23665
} 


\section{Introduction}

The electron beam ion trap (EBIT) at the Lawrence Livermore National Laboratory was specifically designed for the study of atomic processes of highly charged ions. ${ }^{1}$ The device provides direct line-of-sight access to the region were an electron beam produces and excites highly charged ions of a particular species of interest, thus allowing for detailed studies of electron-ion interactions with spectroscopic means. This feature makes EBIT unique among the present generation of atomic physics facilities, including heavy-ion storage rings, electron beam ion sources (EBIS), and electron cyclotron resonance (ECR) sources. ${ }^{2}$ The low-energy version of EBIT employs electrons with energy as high as $30 \mathrm{keV}$ and allows the study of bare molybdenum. Recently, a highenergy version of EBIT, dubbed SuperEBIT, was completed. ${ }^{3}$ It employs electron beams with energies as high as $200 \mathrm{keV}$ to produce fully stripped uranium ions.

Because of the high charge states involved our spectroscopy efforts are concentrated on analyzing radiation in the $\mathrm{x}$-ray regime. A variety of high-resolution flatcrystal and curved-crystal spectrometers are available for this purpose ${ }^{4}$ providing the necessary degree of flexibility in matching resolving power and throughput with the requirements of a given measurement. The range of $x$-ray energies spanned by these instrument presently extends from 650 to $15,000 \mathrm{eV}$. The high-resolution crystal spectrometers are complemented with a pulse-height analysis system utilizing high-purity germanium or $\mathrm{Si}(\mathrm{Li})$ detectors and providing a broad-band analysis of the $\mathrm{x}$-ray emission from EBIT from 0.5 to $350 \mathrm{keV}$.

Our current effort in $\mathrm{x}$-ray spectroscopy on the EBIT facility provides information that can be divided into three general categories:

(1) Position information on the spectral lines. It allows the precise measurement of the energy levels. Such measurements are crucial for line identification and line blends, as well as tests of relativity and quantum electrodynamics.

(2) Intensity information on the spectral lines. It allows the measurement of the electron-ion interaction cross sections. The cross section measurements include electronimpact excitation, dielectric recombination, innershell ionization, and resonance excitation. The intensity information also allows the study of polarization phenomena.

(3) The temporal evolution of the spectral lines. It allows the study of transient phenomena such as innershell ionization and line emission by radiative electron capture. It also allows the study of radiative lifetimes of long-lived excited states.

The electron beam energy provides yet another dimension to the range of experimental conditions EBIT can access. The beam is quasi mono-energetic with an energy spread of 30-50 eV FWHM and can be changed at a rate of $10 \mathrm{~V} / \mu$ s, i.e., at a rate much faster than that of any electron-ion interaction process. As a result, we can use EBIT to make an ion at one energy and probe it with electrons at a second energy, 
selecting the electron-ion interaction process of interest. The line formation processes in EBIT are thus very different from those in a plasma, where virtually all processes occur simultaneously.

The atomic physics issues investigated with EBIT include the ionization of the lithiumlike transition metals, ${ }^{5}$ electron-impact excitation cross-sections of heliumlike titanium, ${ }^{6}$ the strength of resonant excitation, ${ }^{7}$ and the cross sections for dielectronic recombination of heliumlike iron, 8 nickel, molybdenum, and barium, ${ }^{9}$ including dielectronic recombination into the high- $n$ Rydberg levels of heliumlike iron. 10 Moreover, a large number of precise line energy measurements have been made. These include measurements along the neonlike and nickellike isoelectronic sequences to determine the line overlap for resonant photo-pumping of $x$-ray lasers, ${ }^{11}$ as well as various measurements to test the predictions of relativity and quantum electrodynamics (QED), such as those in sodiumlike platinum and neonlike ytterbium. ${ }^{12}$ Very recently we also made the first measurement of a long-lived (about $100 \mu \mathrm{s}$ ) metastable level in a highly charged ion by studying the radiative decay of the $1 \mathrm{~s} 2 \mathrm{~s}{ }^{3} \mathrm{~S}_{1}$ level in heliumlike neon. 13

Our current measurements on EBIT focus on developing a complete understanding of the $n=2$ to $n=1 \mathrm{~K}$-shell $\mathrm{x}$-ray spectrum of heliumlike krypton, including its satellite spectrum. The K-shell krypton spectrum may play an important role in the diagnostics of future tokamaks, such as the International Tokamak Engineering Reactor (ITER), which are predicted to be much hotter than current machines. Our effort also includes the development of techniques to simulate and to study the atomic processes dominant in ionizing and recombining plasmas, such as those found in shock-heated super nova remnants or in photo-ionized nebulae. These studies involve both the 1-2 Kshell and 2-3 L-shell emission of iron. Our tests of relativity and QED currently concentrate on studying the $x$-ray emission from lithiumlike uranium, which was made possible by the advent of SuperEBIT. Curiously enough, the measurements of lithiumlike uranium benefitted greatly by the understanding gained from the study of ionization and recombination effects in the medium- $\mathrm{Z}$ ions, as shown by our discussion below.

\section{K-shell Spectrum Of Krypton}

It is anticipated that future, ignition-type magnetic fusion plasmas will have electron temperatures between 10 and $30 \mathrm{keV}$. At these temperatures, low- $Z$ and medium- $Z$ ions, such as argon, titanium, or iron, that serve as ion temperature diagnostics in existing tokamaks, will be fully stripped at the core of the plasma. As a result, an iontemperature diagnostic based on Doppler-broadening measurements of K-shell $\mathrm{x}$-ray lines from the much heavier noble gas krypton has been proposed to monitor the hot plasmas 
of ITER. ${ }^{14}$ The ionization potential of lithiumlike krypton is $4.8 \mathrm{keV}$, that of heliumlike krypton is $17.8 \mathrm{keV}$. Hence the heliumlike ion will exist at the plasma core for temperatures in excess of $25 \mathrm{keV}$ before burning out. By contrast, the ionization potentials of lithiumlike and heliumlike iron are 2.1 and $8.8 \mathrm{keV}$. An ion temperature diagnostic based on the krypton $\mathrm{K}$-shell lines has an additional advantage that the radiation involved is in excess of $13 \mathrm{keV}$. This is twice the energy of the $\mathrm{K}$-shell $\mathrm{x}$ rays from iron. The vacuum window separating the spectrometer and the plasma can thus be made much thicker than windows currently in use on spectrometers monitoring the emission from the transition metals. For example, a 5-mm thick beryllium window has a $70 \%$ transmission rate for the krypton $\mathrm{K}$-shell $\mathrm{x}$ rays.

To ensure the reliability of the Doppler-broadening measurements, it is important to identify suitable lines free of blends. Measurements on present-day tokamaks employ iron or nickel and focus on the heliumlike resonance line $1 \mathrm{~s} 2 \mathrm{p}{ }^{1} \mathrm{P}_{1} \rightarrow 1 \mathrm{~s}^{2}{ }^{1} \mathrm{~S}_{0}$, labeled $w$. Recent measurements of the K-shell krypton spectrum on the TFTR tokamak, however, have indicated the possibility of utilizing the forbidden line $1 \mathrm{~s} 2 \mathrm{~s}^{3} \mathrm{~S}_{1} \rightarrow 1 \mathrm{~s}^{2}{ }^{1} \mathrm{~S}_{0}$, labeled $z$, as the other lines were thought to be largely contaminated by satellites. ${ }^{15}$ Understanding the krypton $\mathrm{K}$ spectrum and choosing the most appropriate line for ion temperature measurements will be the more crucial for ITER than for present-day applications because $x$-ray spectroscopy may represent the only means of determining the ion temperature. Other techniques in wide use today, such as charge-exchange recombination spectroscopy, may fail because of the large plasma volume and small beam penetration depths.

Our study of krypton illustrates the techniques available to us on EBIT. In Fig. 1 we show spectra of the $\mathrm{K}$-shell emission observed at four different electron beam energies with our high-resolution von Hámos-type crystal spectrometer. The energies were chosen so as to excite distinct sets of dielectronic resonances: the 1s212l' KLL resonances centered at about $9 \mathrm{keV}$ are shown in Fig. 1 (a), and the 1s213l' KLM resonances near $11.4 \mathrm{keV}$ are shown in Fig. 1 (b). A spectrum obtained by sweeping the beam energy over all of the $1 \mathrm{~s} 2 \mathrm{lnl}$ ' resonances above KLM, i.e., the resonances with $n \geq 4$ that fall into the range $12.4-13.1 \mathrm{keV}$ are shown in Fig. 1 (c). A spectrum of the lines formed by direct electron-impact excitation displaying the heliumlike lines and their collisional satellite lines is given in Fig. 1 (d). This spectrum was obtained at energies near $14.5 \mathrm{keV}$, i.e. above the threshold for direct excitation. Measurements were carried out in second order Bragg reflection employing a $\mathrm{LiF}(200)$ crystal bent to a $75-\mathrm{cm}$ radius of curvature and a position-sensitive proportional counter with a $3 \times 10 \times 0.4 \mathrm{~cm}^{3}$ active volume. The counting gas was a mixture of $70 \%$ xenon and $30 \%$ methane at a 78 psig.

We are conducting a detailed analysis of the spectra, which includes a comprehensive identification of all lines observed, a precise determination of their 
spectral positions, and a measurement of their excitation cross sections. Although this analysis is still in progress, we can note several results.

The EBIT measurements confirm the contention by Bitter et al. ${ }^{15}$ that the line labeled $z$ is the least affected by blends with satellite transitions and thus may be among the best lines to use for ion temperature measurements. It overlaps neither with dielectronic nor with collisional satellite transitions. By contrast, the heliumlike resonance line $w$ blends with a strong contingent of dielectronic satellite transitions involving spectator electrons in the $n \geq 3$ shells. These types of satellite lines are already strong in the spectra of the heliumlike transition metals. ${ }^{16}$ Their intensity increases further in accordance with the $\mathrm{Z}^{4}$ scaling of their radiative transition rates, and in krypton they cause a significant broadening and shift of the apparent line $w$. Their presence must be accounted for in a detailed fashion, if line $w$ is to be used for ion temperature measurements. In a measurement on TFTR, Bitter et al. found, for example, that the temperature inferred from the apparent line $w$ was more than twice the value inferred from line $z .{ }^{15}$ Using the EBIT measurements we can determine both the exact positions and the intensities of the high-n dielectronic satellite lines that blend with $w$. With this information it will become possible to construct synthetic spectra that allow modeling of the apparent resonance line $w$ and to accurately determine the plasma temperature despite the blends.

\section{Measurements of Transient Ionization Phenomena}

A large class of hot plasmas exists in which the plasma parameters are changing on time scales faster than required to establish ionization equilibrium. For example, rapid heating by shock compression of young supernova remnants may result in a plasma that is underionized relative to the local electron temperature. In such a case, a K-shell emission spectrum shows an excess of lower-energy $x$ rays relative to the emission expected under the assumption of ionization equilibrium, as shown in various studies of iron by Mewe et al. ${ }^{17}$. The sudden temperature rise strongly favors ionization and recombination is suppressed. Thus spectral features associated with recombination, such as dielectronic satellite transitions, are virtually absent. The K-shell emission features instead are produced by electron-impact excitation and innershell ionization, i.e., the removal of a $1 \mathrm{~s}$ electron

$$
1 \mathrm{~s}^{2} 2 \mathrm{l}^{\mathrm{m}}+\mathrm{e}^{-}->1 \mathrm{~s} 2 \mathrm{l}^{\mathrm{m}}+2 \mathrm{e}^{-}
$$

The latter process is of particular importance, as it leaves ions in excited states that are in many instances rarely accessed by electron-impact excitation, and thus provides the signature of an ionizing plasma. 
Iron ions embedded in a solar flare with an electron density of $10^{11} \mathrm{~cm}^{-3}$ need more than $10 \mathrm{~s}$ to reach ionization equilibrium, if the temperature jumps from 500 to $5000 \mathrm{eV}$, as shown by Mewe et al. ${ }^{17}$ The same ions may need about 3000 years to reach ionization equilibrium in a supernova remnant whose density is $10 \mathrm{~cm}^{-3}$. In EBIT with an electron density of about $10^{12} \mathrm{~cm}^{-3}$ equilibrium is reached within about 1 second. Despite the large differences in the absolute time scales, the evolution of the K-shell emission spectra is the same for each case provided the time is scaled by the electron density.

The time sequence of the K-shell emission from ionizing iron ions was measured on EBIT by using the following procedure. Near neutral iron ions $\left(\mathrm{Fe}^{1+}\right.$ and $\left.\mathrm{Fe}^{2+}\right)$ are injected into EBIT at $t=0 \mathrm{~s}$. Then a set of 15 spectra are recorded, each integrating the emission for a fixed length of time, which varied from 7 to $20 \mathrm{~ms}$. After $300 \mathrm{~ms}$ the trap is emptied, and the sequence restarted. The threshold for impact excitation of K-shell $\mathrm{x}$ rays is about $6.7 \mathrm{keV}$, that for innershell ionization is $8.68 \mathrm{keV}$. To study both effects, we measured the time sequence for beam energies of $6.9 \mathrm{keV}$ and $12.0 \mathrm{keV}$. Three representative spectra for the $12.0 \mathrm{keV}$ case are shown in Fig. 2.

A comparison of the EBIT measurements with the predictions by Mewe et al. ${ }^{17}$ shows strikingly good agreement. This agreement is achieved despite the fact that the predictions by Mewe et al. 17 assume a Maxwellian electron temperature, while the excitation in EBIT relies on a monoenergetic beam of electrons. The reasons are that even in a plasma only those electrons contribute to the excitation that have energies larger than threshold. These electrons are located in tive tail of the distribution, and their effect in a first approximation can be modeled well by our monoenergetic beam.

Lines predominantly excited by innershell ionization are labeled in the figure. These lines provide markers for the transient ionization and are thus important for diagnostic applications and the proper interpretation of the data. The forbidden heliumlike line $1 \mathrm{~s} 2 \mathrm{~s}{ }^{3} \mathrm{~S}_{1} \rightarrow 1 \mathrm{~s}^{2}{ }^{1} \mathrm{~S}_{0}$, labeled $z$, represents such a marker. Through innershell ionization its intensity is strongly coupled to the abundance of lithiumlike ions, provided the electron energy is above the innershell ionization threshold. This is illustrated in Fig. 3, which shows a great enhancement of line $z$ in the presence of a large abundance of lithiumlike ions. A study of the intensity of a line produced by innershell ionization as the ionization balance is varied can yield a measure of the ionization cross section. 18 Such a study was carried out for line $z$ and provided the innershell ionization cross section of lithiumlike chromium and iron ions. ${ }^{19}$ A similar determination is in progress for the innershell innization cross sections of the lower-charge iron ions. Together with data for the electron-impact excitation, our transient-ionization measurements will provide detailed tests of the theoretical predictions of K-shell line formation in transient, underionized plasmas. 


\section{Measurements of Transient Recombination Phenomena}

\section{CEIVE

The intense radiation field accompanying such cosmic objects as $\mathrm{x}$-ray binaries may result in the ionization of the surrounding medium to stages that exceed by far those expected from the plasma temperature. Line formation in such sources occurs not by collisional excitation but by radiative and dielectronic recombination. For example, Liedahl et al. ${ }^{20}$ considered L-shell line formation from few-electron iron ions embedded in a photoionized nebula with an electron temperature of $10 \mathrm{eV}$. Like in the case of transient ionization, where the plasma is underionized relative to the local electron temperature, the spectra from recombining sources where the plasma is overionized relative to the local temperature, differ drastically from those produced in collisional equilibrium.

We have begun to study line emission on EBIT under conditions that are similar to those found in overionized, recombining plasma. This is done by making use of the capability to switch the electron beam rapidly from one value to another, and thus to produce the ions at one energy and to study them at another. In particular, we produced hydrogenic iron ions at a beam energy of $12 \mathrm{keV}$; then we switched the beam to $5.5 \mathrm{keV}$, i.e., to a value well below the threshold for collisional excitation, and monitored the $x$ rays produced by the recombination process. The only recombination process allowed at this energy is radiative electron capture.

A comparison of the $\mathrm{K}$-shell iron spectra obtained at the two energies is given in Fig. 4. The spectrum produced by electron capture differs markedly from the collisionally excited spectrum. While the emission in the collisional spectrum is dominated by the singlet line, it is dominated by the forbidden triplet line in the recombination spectrum. Electron-impact excitation produces collisional satellite lines, when ions in a lower charge state than heliumlike are present. By contrast, in a recombination-produced spectrum the four heliumlike lines are the only lines that can be excited, and no satellites may be present.

Predictions of recombination-produced K-shell spectra were made by Scofield. ${ }^{21}$ Our measurements are in qualitative agreement with these predictions, and a quantitative analysis is in progress.

\section{Measurements of 2-2 Transitions in $\mathrm{U}^{82+}$ through $\mathrm{U}^{89+}$}

Energy shifts due to quantum electrodynamical effects increase as $Z^{4}$. Among the naturally occuring elements the effects are thus largest in uranium. The QED-induced energy shift of the $2 \mathrm{~s}_{1 / 2}$ level in lithiumlike uranium $\mathrm{U}^{89+}$ is more than $47 \mathrm{eV}$. Similar values are found for the $2 s_{1 / 2}$ levels in the neighboring charge states. This shift represents a large fraction of the overall $2 s_{1 / 2}-2 p_{1 / 2}$ or $2 s_{1 / 2}-2 p_{3 / 2}$ transition energies, and 
a precise measurement of these transitions is a sensitive test of the predictions of QED theory. Using SuperEBIT we have made a measurement of thirteen $2 \mathrm{~s}_{1 / 2}-2 \mathrm{p}_{3 / 2}$ transitions in the eight ionization states $\mathrm{U}^{82+}$ through $\mathrm{U}^{89+}$ achieving an accuracy of about $0.3 \mathrm{eV}$, and complete agreement with predicted QED values was found. ${ }^{22}$

Because of the small number of possible electric dipole (E1) transitions involved the $2 s_{1 / 2}-2 p_{3 / 2}$ spectrum from fluorinelike through lithiumlike uranium ions is relatively easy to interpret. By looking at all excited configurations involving the $n=2$ shell we find, for example, that the lithiumlike, berylliumlike, carbonlike, and fluorinelike ions have exactly one possible $2 s_{1 / 2}-2 p_{3 / 2}$ E1 transition. The other ions have more, but the situation is only slightly more complicated. Looking at the levels involved and relying on the selection rules for dipole transitions we were able to identify all lines observed in our measurements. A typical spectrum is shown in Fig. 5(a).

Using the same approach we also made a prediction of the $2 p_{1 / 2}-2 p_{3 / 2}$ magnetic dipole (M1) spectrum. The $2 \mathrm{p}_{1 / 2}-2 \mathrm{p}_{3} / 2 \mathrm{M} 1$ transitions are situated around $3900 \mathrm{eV}$, i.e., in a region about $600 \mathrm{eV}$ below that of the $\mathrm{E} 1$ transitions. Here we assume that levels that can decay via an $\mathrm{E} 1$ transition will do so and do not produce a magnetic dipole line. This reduces the number of possible $M 1$ lines, and, as a result, no $2 p_{1 / 2}-2 p_{3 / 2}$ line is produced in the lithiumlike ion. A measurement of the $2 p_{1 / 2}-2 p_{3 / 2}$ magnetic dipole spectrum is shown in Fig. 5(b). Again, using simple considerations we identified all but one observed transition. The one additional transition observed can be readily identified as a $2 p_{1 / 2}-2 p_{3 / 2}$ electric quadrupole (E2) transition in the carbonlike ion. It proceeds from the $\left(2 \mathrm{~s}_{1 / 2} 2 \mathrm{p}_{1 / 2}^{2} \mathrm{p}_{3 / 2}\right)_{\mathrm{J}=2}$ level, which is the lowest excited level in the carbonlike ion and which has no other decay channel open to it but radiative decay to the $\mathrm{J}=0$ ground level.

To confirm our line identifications we constructed a detailed collisional-radiative model constructed from data generated with the Hebrew University-Lawrence Livermore atomic structure codes (HULLAC). ${ }^{23}$ Including all excited levels in the $n=2$ shell, we used the model to predict the relative line intensities from a balance of all radiative transitions and electron-impact collisions connecting the $n=2$ excited levels to each other and to the ground state. Results from these calculations are shown in Fig. 6. The calculations accurately predicted the intensities of the E1 transitions. They did not, however, accurately predict the relative intensities of the M1 transitions. These were predicted much too small relative to that of the El lines. If the predictions had been correct, we would have never been able to measure the magnetic dipole lines given the background from bremstrahlung and cosmic ray events.

The discussion in the preceeding two sections has shown that innershell ionization and radiative electron capture can play a dominant role in line formation. This is true especially for the dipole-forbidden lines, as demonstrated for the $1 \mathrm{~s} 2 \mathrm{~s}{ }^{3} \mathrm{~S}_{1} \rightarrow 1 \mathrm{~s}^{2}{ }^{1} \mathrm{~S}_{0}$ magnetic dipole line in the heliumlike ion. As a result, we expect that such processes 
also play a major role in the excitation of the magnetic dipole spectrum of uranium. For example, ionization of a $2 \mathrm{p}_{1 / 2}$ electron from the nitrogenlike ion populates the levels $\left(2 \mathrm{~s}^{2} 2 \mathrm{p}_{1 / 2} 2 \mathrm{p}_{3 / 2}\right)_{\mathrm{J}=1,2}$ in the carbonlike ion and leads to the excitation of both a magnetic dipole and an electric quadrupole line.

To predict the effects of radiative electron capture and of ionization we expanded our collisional-radiative model. We included radiative electron capture into the $n=2$ and $n=3$ shells and ionization of $n=2$ electrons. This links a given ion species to its neighboring ion species. We also included all radiative transitions connecting levels with an $n=3$ optical electron to the $n=2$ excited states and ground as well as all collisonal processes. The results of these calculations are shown in Fig. 6. While little change in the intensities of the $2 s_{1 / 2}-2 p_{3 / 2}$ electric dipole transitions is found, we note a large increase of the intensity of $2 \mathrm{p}_{1 / 2}-2 \mathrm{p}_{3 / 2}$ magnetic dipole spectrum, resulting in a good agreement with the observed intensities.

The calculations based on the expanded radiative-collisional model show that ionization, electron capture, and radiative cascades from the $n=3$ shell contribute each roughly one third of the total excitation flux, while direct electron-impact excitation from the ground level is nearly negligible. The comparison between our measurements and the predictions from our radiative-collisional model confirms the importance of ionization and recombination for the excitation of dipole-forbidden lines. In fact, the predictions still seem to underestimate the actual strength of the magnetic dipole lines suggesting that additional indirect excitation processes, such as radiative cascades from and electron capture into the $n \geq 4$ shells, play an important role as well.

Using the collisional-radiative model we have also been able to predict the transitions from neonlike uranium. Unlike those in the lithiumlike through fluorinelike ions, the intensity of the $2-2$ transitions in neonlike ions cannot be predicted by simply looking at the possible transitions between excited levels because of the complicating presence of a spectator electron in the $n=3$ shell. The collisional-radiative model, expanded to include ionization of sodiumlike ions and recombination from fluorinelike ions, predicts a multitude of neonlike transitions. Most of these lines, however, are very weak. The predictions are again confirmed by our observations. In our measurement, we were able to observe only one neonlike transition, namely the transition predicted to be strongest (cf. Fig. 5).

Comparing our measured transition energies to theory we find that standard multi-configuration Dirac-Fock (MCDF) calculations significantly overestimate the transition energies, as illustrated in Fig. 7. The discrepancy increases with the number of spectator electrons in the $n=2$ shell and can be attributed to residual correlation energies unaccounted for by the atomic structure calculations. There is a distinct difference, however, in the way magnetic dipole transitions differ from theory compared to electric dipole transitions. This is caused by the fact that QED effects are small for $2 p$ electrons 
relative to those for $2 \mathrm{~s}$ electrons. Unlike the $2 \mathrm{~s}_{1 / 2}-2 \mathrm{p}_{3 / 2}$ transitions, the $2 \mathrm{p}_{1 / 2}-2 \mathrm{p}_{3 / 2}$ transitiors are therefore not greatly affected by QED effects. The greater deviation of the E1 transitions from theory thus reflects the additional error introduced into the calculations by quantum electrodynamics.

\section{Conclusion}

The EBIT and SuperEBIT facilities are now able to produce any ion up to bare uranium, allowing us to measure transition energies as well as line-formation processes along complete isoelelectronic sequences. These measurements provide benchmarks for the development of atomic structure theories and codes, test the predictions of QED theory, and calibrate detailed collisional-radiative models used in spectral modeling and interpretation.

The conditions in EBIT are similar to those in low-density plasmas such as tokamaks and astrophysical sources. EBIT can thus be used to develop spectral diagnostics applicable to the investigation of such sources. One example is our measurement of the K-shell $\mathrm{x}$-ray satellite spectrum of heliumlike krypton, which may play an important role in the diagnostics of future tokamaks such as ITER.

In this short overview, we have not been able to discuss the development and implementation of various experimental refinements and new techniques. One of these is the development of very high resolution spectrometers $(\lambda \Delta \lambda \geq 5,000)$, which take full advantage of the fact that the ions in EBIT are virtually at rest with a temperature predicted to be less than a few hundred $\mathrm{eV}$. These techniques promise to open entirely new areas for atomic physics studies on EBIT.

This work was supported in part by the Office of Fusion Energy and NASA grant No. NAGW-2688 and was performed at Lawrence Livermore National Laboratory under the auspices of the U.S. Department of Energy under contract No. W-7405-ENG-48. 


\section{References}

1. M. A. Levine, R. E. Marrs, J. R. Henderson, D. A. Knapp, and M. B. Schneider, Phys. Scr. T22, 157 (1988); R. E. Marrs, M. A. Levine, D. A. Knapp, ind J. R. Henderson, Phys. Rev. Lett. 60, 1715 (1988).

2. R. E. Marrs, Comments At. Mol. Phys. 27, 57 (1991).

3. D. A. Knapp, R. E. Marrs, S. R. Elliott, E. W. Magee, and R. Zasadzinski, Nucl. Instrum. Methods A334, 305 (1993).

4. P. Beiersdorfer, R. E. Marrs, J. R. Henderson, D. A. Knapp, M. A. Levine, D. B. Platt, M. B. Schneider, D. A. Vogel, and K. L. Wong, Rev. Sci. Instrum. 61, 2338 (1990); P. Beiersdorfer, Nucl. Instrum. Methods B56/57, 1144 (1991); P. Beiersdorfer and B. J. Wargelin, Rev. Sci. Instrum. (in press).

5. K. Wong, P. Beiersdorfer, M. H. Chen, R. E. Marrs, K. J. Reed, J. H. Scofield, D. A. Vogel, and R. Zasadzinski, Phys. Rev. A 48, 2850 (1993).

6. S. Chantrenne, P. Beiersdorfer, R. Cauble, and M. B. Schneider, Phys. Rev. Lett. 69, 265 (1992).

7. P. Beiersdorfer, A. L. Osterheld, M. H. Chen, J. R. Henderson, D. A. Knapp, M. A. Levine, R. E. Marrs, K. J. Reed, M. B. Schneider, and D. A. Vogel, Phys. Rev. Lett. 65, 1995 (1990).

8. P. Beiersdorfer, T. W. Phillips, K. L. Wong, R. E. Marrs, and D. A. Vogel, Phys. Rev. A 46, 3812 (1992).

9. D. A. Knapp, R. E. Marrs, M. B. Schneider, M. H. Chen, M. A. Levine, and P. Lee, Phys. Rev. A 47, 2039 (1993).

10. P. Beiersdorfer, M. B. Schneider, M. Bitter, and S. von Goeler, Rev. Sci. Instrum. 63, 5029 (1992).

11. P. Beiersdorfer, J. Nilsen, A. Osterheld, D. Vogel, K. Wong, R. E. Marrs, and R. Zasadzinski, Phys. Rev. A 46, R25 (1992); S. Elliott, P. Beiersdorfer, and J. Nilsen, Phys. Rev. A 47, 1403 (1993); S. R. Elliott, P. Beiersdorfer, and J. Nilsen, in VIth International Conference on the Physics of Highly Charged Ions, AIP Conference Proceedings No. 274, ed. by P. Richard, M. Stöckli, C. L. Cocke, and C. D. Lin (AIP, New York, 1993), p. 556.

12. T. E. Cowan, C. Bennett, D. Dietrich, J. V. Bixler, C. J. Hailey, J. R. Henderson, D. A. Knapp, M. A. Levine, R. E. Marrs, and M. B. Schneider, Phys. Rev. Lett. 66, 1150 (1991); P. Beiersdorfer, M. H. Chen, R. E. Marrs, and M. A. Levine, Phys. Rev. A 41, 3453 (1990).

13. B. J. Wargelin, P. Beiersdorfer, and S. M. Kahn, Phys. Rev. Lett. 71, 2196 (1993).

14. M. Bitter, H. Hsuan, K. W. Hill, R. Hulse, M. Zarnstorff, and P. Beiersdorfer, in Atomic and Plasma-Material Interaction Processes in Controlled Thermonuciear Fusion, edited by R. K. Janev and H. W. Drawin (Elsevier), p. 119-133. 
15. M. Bitter, H. Hsuan, C. Bush, S. Cohen, C. J. Cummings, B. Grek, K. W. Hill, J. Schivell, M. Zarnstorff, P. Beiersdorfer, A. Osterheld, A. Smith, and B. Fraenkel, Phys. Rev. Lett. 71, 1007 (1993).

16. V. Decaux, M. Bitter, H. Hsuan, K. W. Hill, S. von Goeler, H. Park, and C. P. Bhalla, Phys. Rev. A 44, R6987 (1991).

17. R. Mewe and J. Schrijver, Astron. Astrophys. 87, 261 (1980); R. Mewe, Phys. Scripta 17, 5 (1984); R. Mewe, J. R. Lemen, G. Peres, J. Schrijver, and S. Serio, Astron. Astrophys. 152, 229 (1985).

18. P. Beiersdorfer, Ph.D. thesis, Princeton University (1988).

19. D. A. Vogel, Ph.D. thesis, Georgia Institute of Technology (1992).

20. D. A. Liedahl, S. M. Kahn, A. L. Osterheld, and W. H. Goldstein, Astrophys. J. 350, L37 (1990).

21. J. H. Scofield, Phys. Rev. A 44139 (1991).

22. P. Beiersdorfer, D. Knapp, R. E. Marrs, S. R. Elliott, and M. H. Chen, Phys. Rev. Lett., in press.

23. A. Bar-Shalom, M. Klapisch, and J. Oreg, Phys. Rev. A 38, 1773 (1988). 


\section{Figures}

Fig. 1. Satellite spectra of heliumlike krypton: (a) $n=2$ dielectronic satellites; (b) $n=3$ dielectronic satellites; (c) $n \geq 4$ dielectronic satellites; (d) collisional satellites. Transitions in heliumlike and lithiumlike krypton are labeled in standard notation. Unlabeled features in (a) and (d) are from berylliumlike and boronlike krypton.

Fig. 2. Transient evolution of the iron K-shell emission after injection into a $12-\mathrm{keV}$ electron beam. Features are labeled by ionization state; lines in heliumlike and lithiumlike iron are labeled in standard notation. Lines excited by innershell ionization are marked with an asterisk.

Fig. 3. Iron K-shell spectra produced with an $18-\mathrm{keV}$ beam in the presence of different relative amounts of lithiumlike target ions. The presence of a large relative density of lithiumlike ions in (b), indicated by the ratio of the lithiumlike line $q$ and heliumlike line $w$, leads to a strong increase in the intensity of the heliumlike line $z$. The increase is caused by innershell ionization of the lithiumlike ions.

Fig. 4. Iron K-shell spectra produced (a) by electron-impact excitation of heliumlike ions at $12 \mathrm{keV}$ and (b) by capture of $5.5-\mathrm{keV}$ electrons by hydrogenic ions.

Fig. 5. Spectra of $2-2$ transitions in highly charged uranium: (a) $2 \mathrm{~s}_{1 / 2}-2 \mathrm{p}_{3 / 2}$ electric transitions; (b) $2 p_{1 / 2}-2 p_{3 / 2}$ magnetic dipole transitions. Lines are labeled by the charge state of the emitting ion. $\mathrm{C}(\mathrm{E} 2)$ denotes an electric quadrupole transition in carbonlike uranium.

Fig. 6. Intensities of the 2-2 transitions in highly charged uranium predicted by a collisional-radiative model. Model (a) includes only electron excitation and radiative cascades among the $n=2$ levels and ground; (b) includes also excitation of and cascades from the $n=3$ levels, as well as excitation by electron capture into excited levels and ionization of $2 \mathrm{~s}$ or $2 \mathrm{p}$ electrons.

Fig. 7. Average difference between MCDF and measured 2-2 transition energies for different charge states of uranium. The difference increases for the lower charge states due to difficulties in calculating the electron correlation terms. The differences are higher for transitions involving the $2 \mathrm{~s}$ subshell because of inaccuracies in calculating the QED terms. Experimental uncertainties are indicated by dashed lines. 


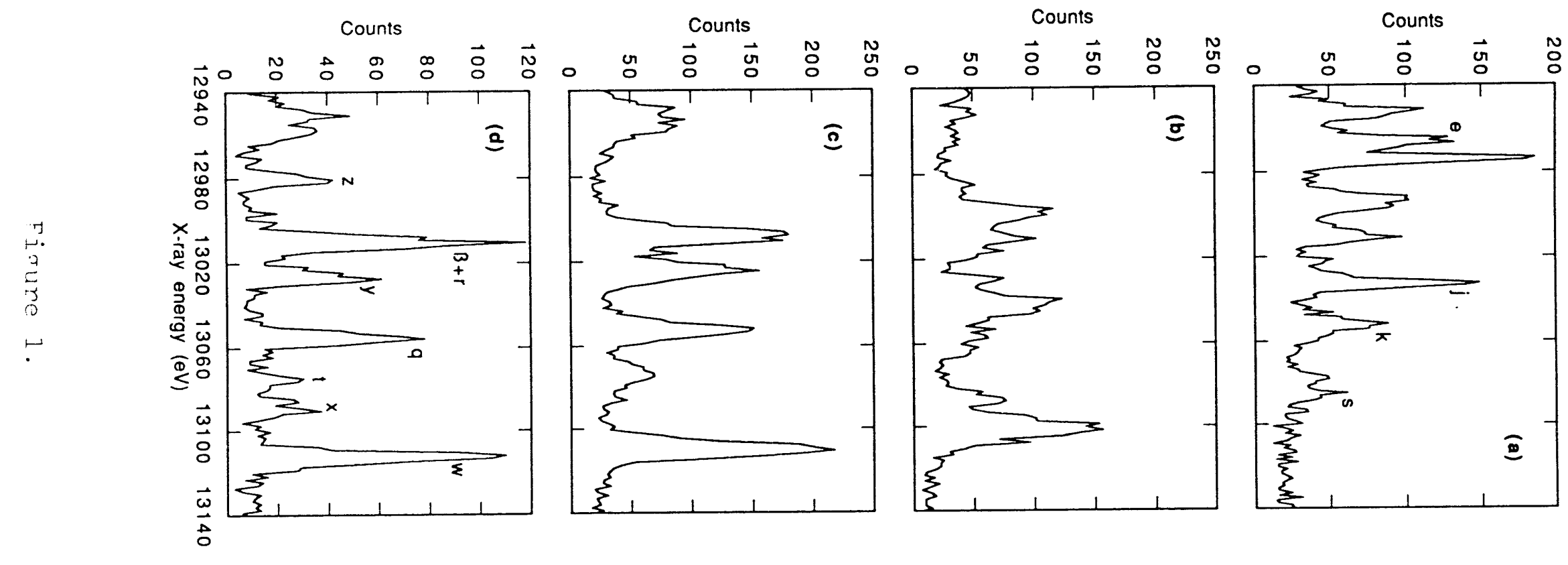



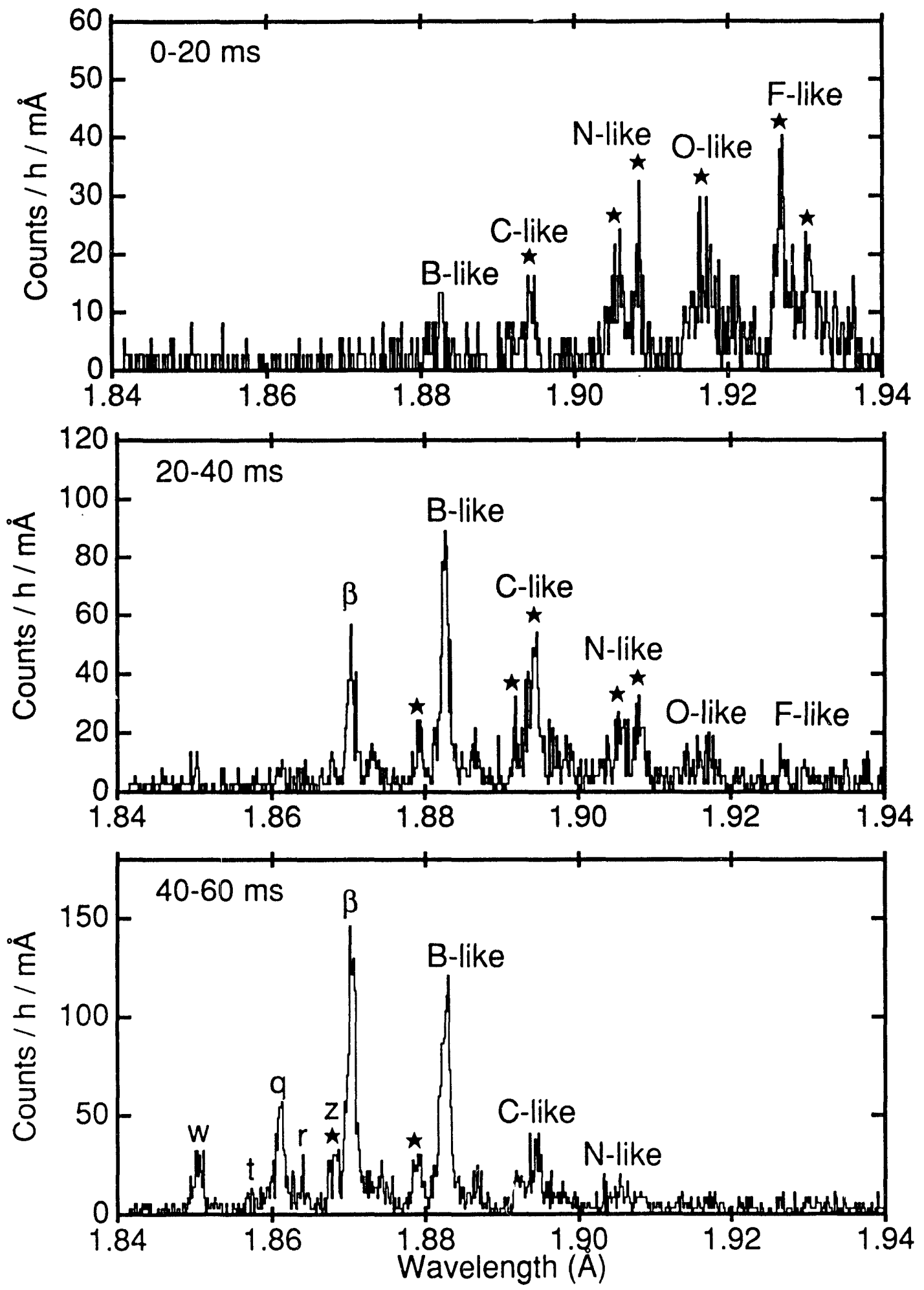


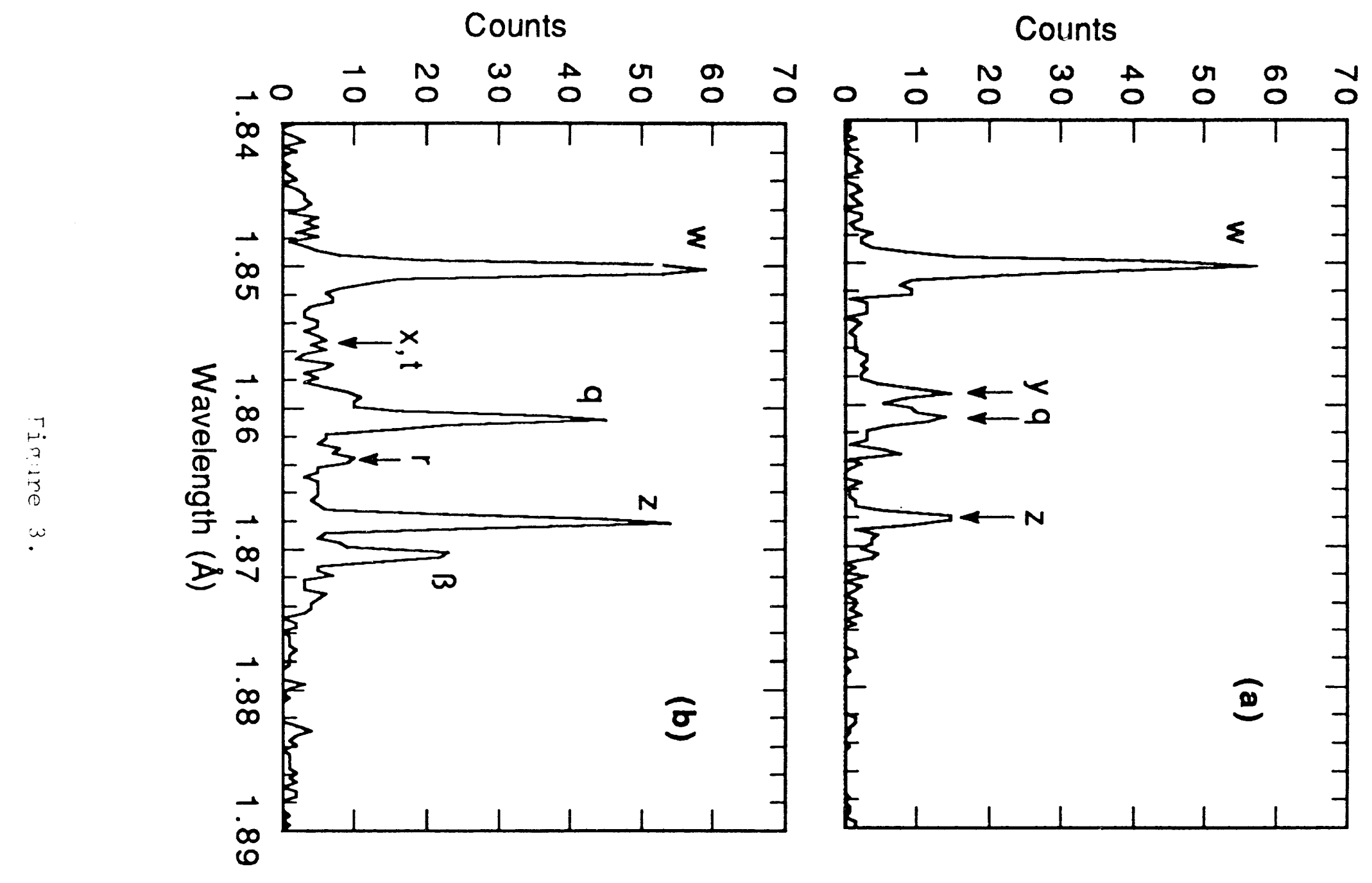




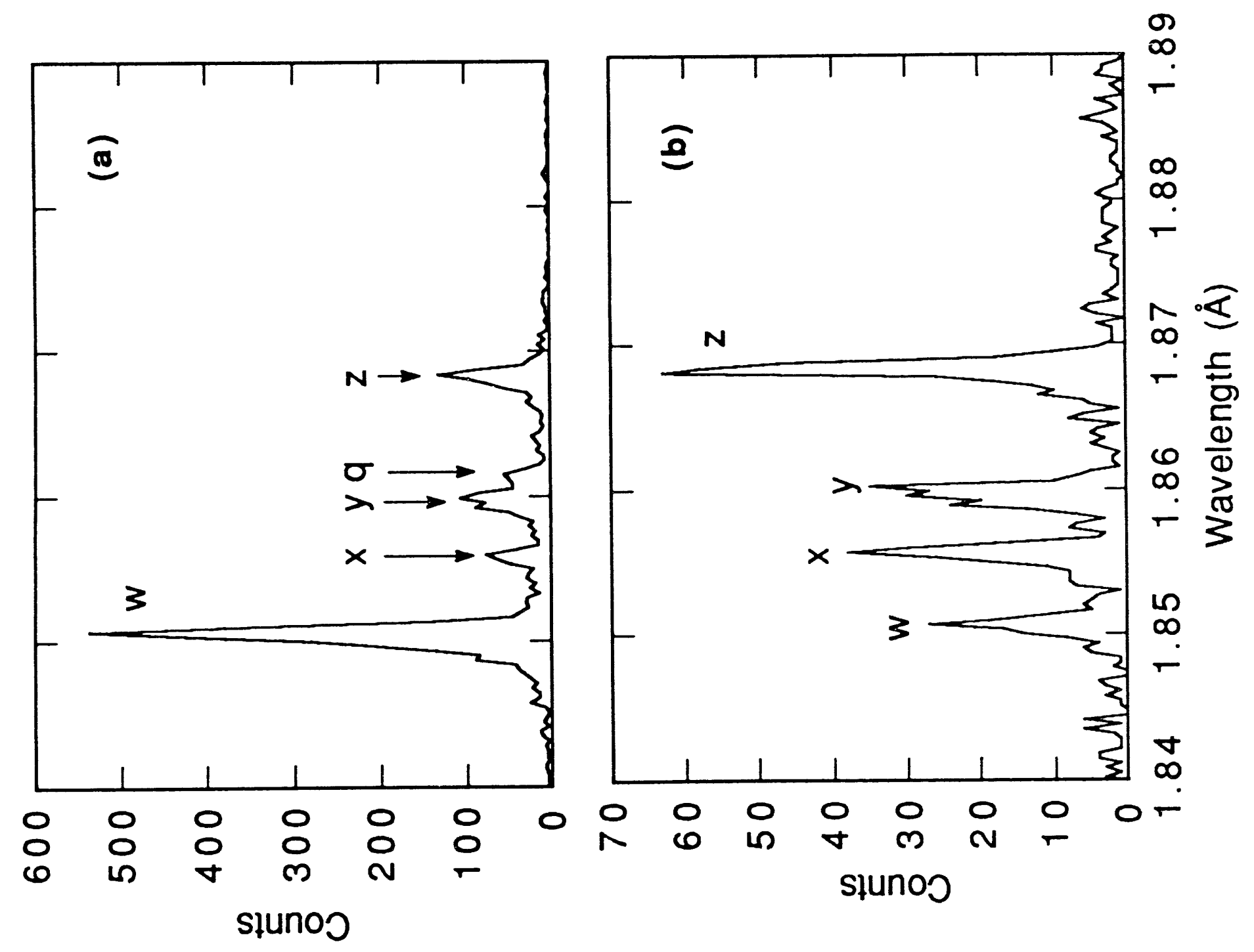



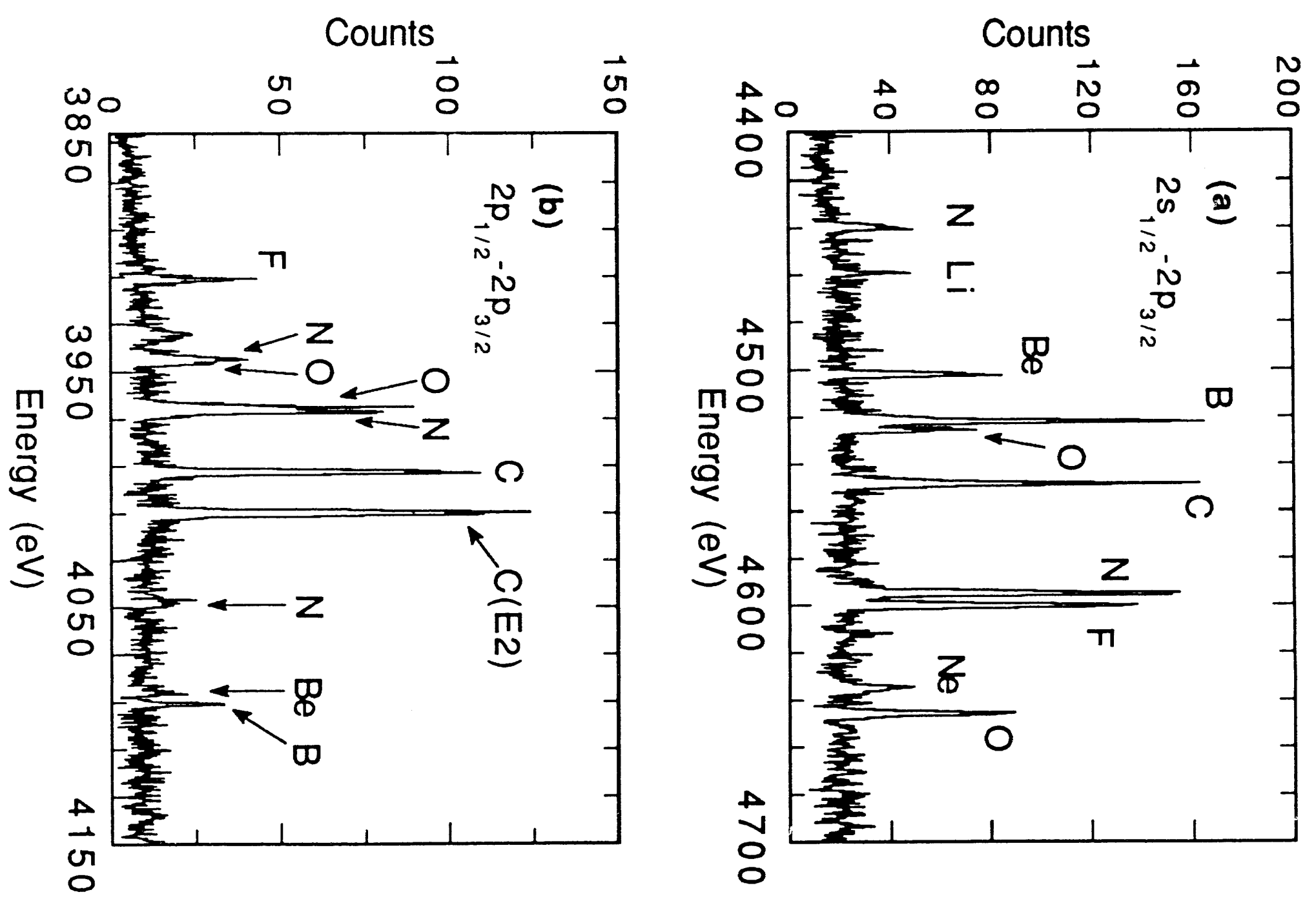

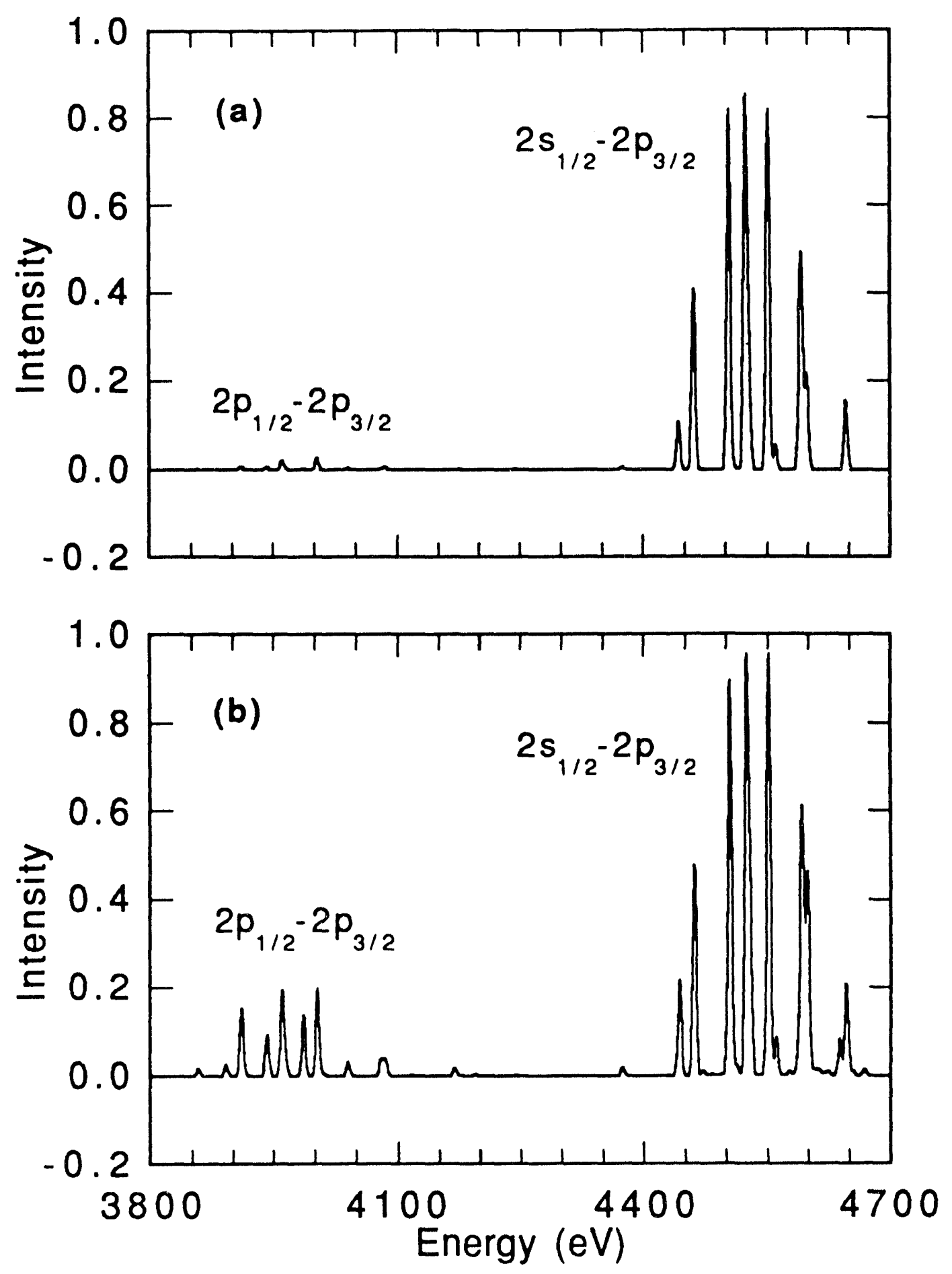

Fioure 6. 


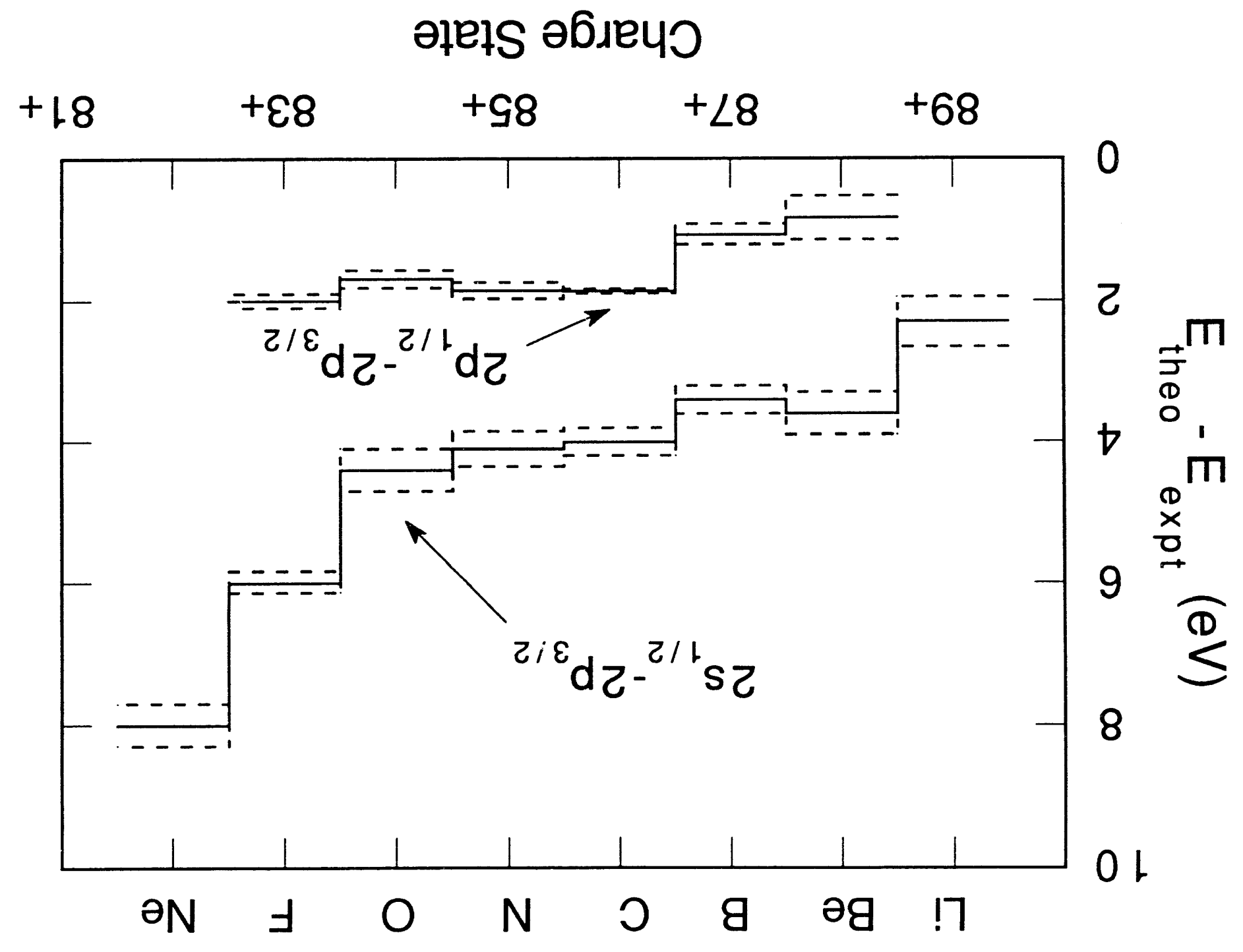



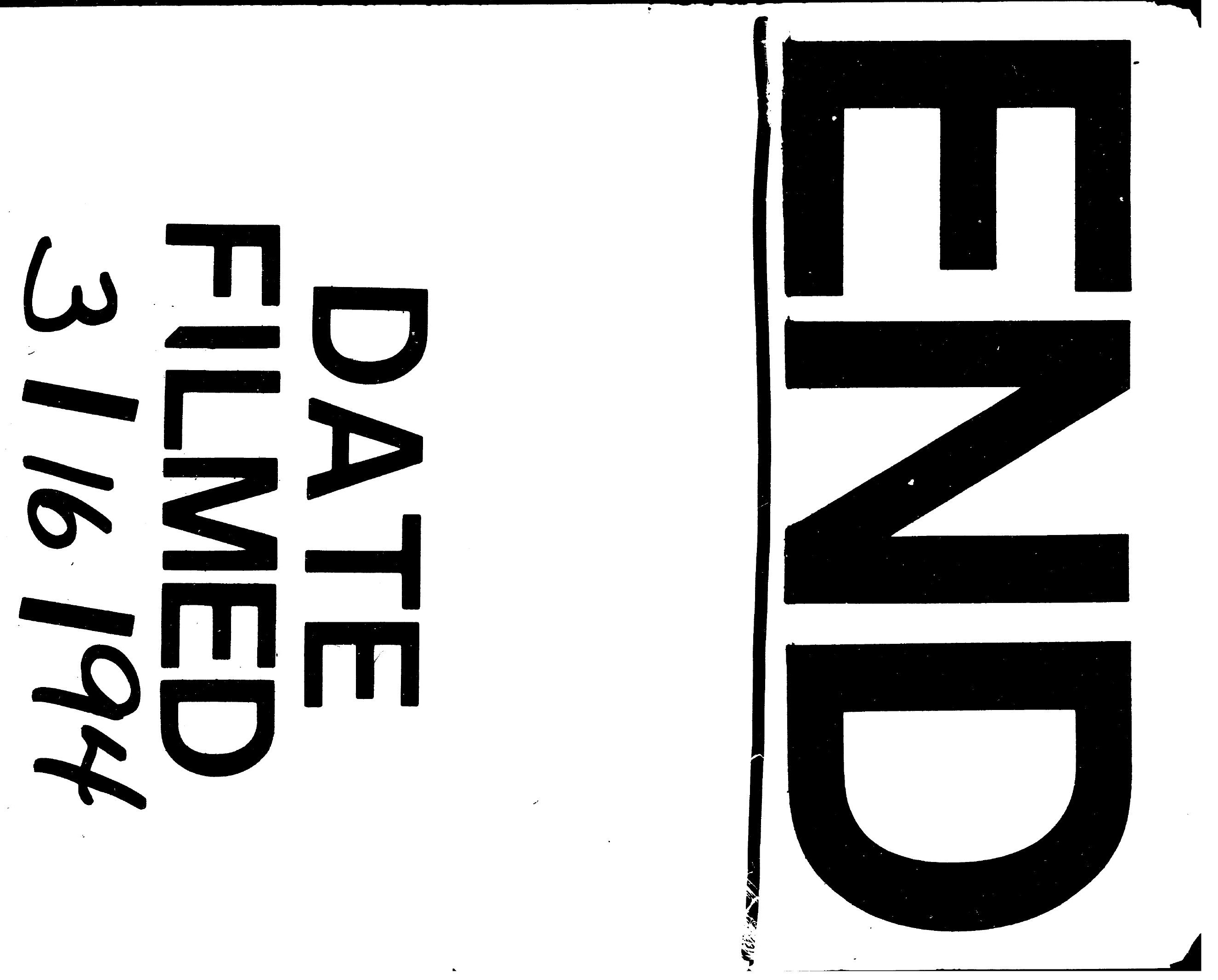


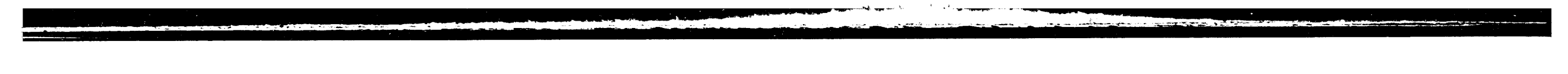
西

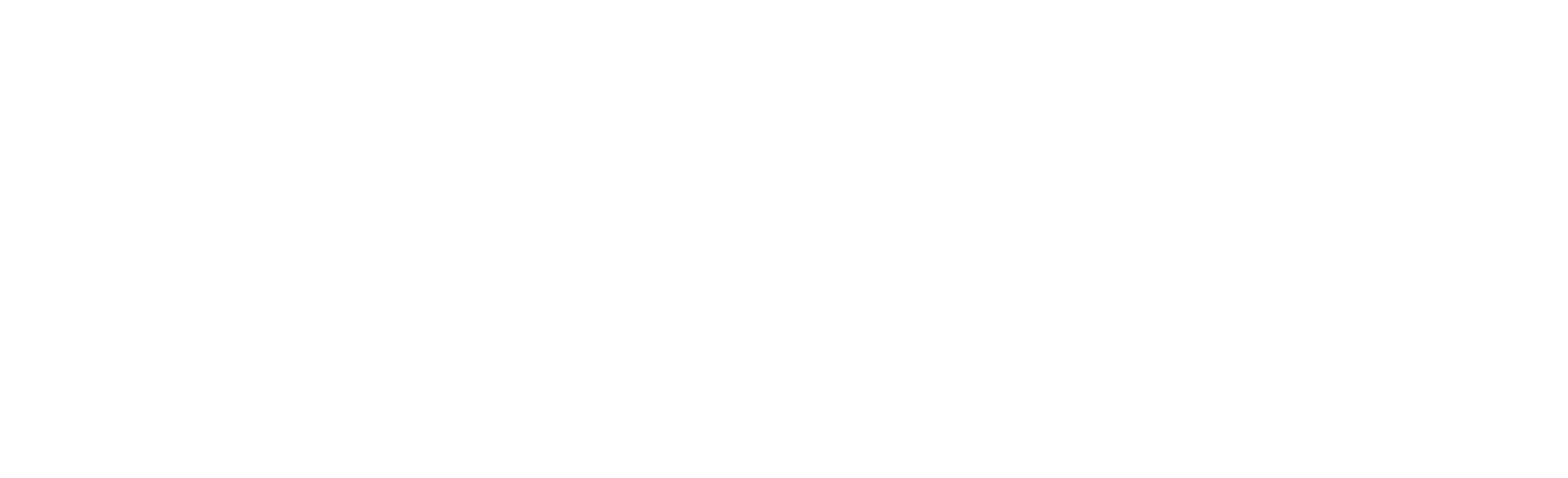

更

